\title{
Challenging the Stigma of Mental Illness: Lessons for Therapists and Advocates. By Patrick W. Corrigan, David Roe, and Hector W.H. Tsang. John Wiley \& Sons Ltd. West Sussex, UK, 2011, 231 pages, $\$ 89.52$
}

\section{Abdul Basit, Ph.D.}

Over the past three decades, the National Alliance on Mental Illness (NAMI) has become increasingly effective; the members of the Association, feeling empowered, have brought about many changes. Not only have they required the mental health professionals to improve the quality of mental health delivery systems, but they have also been able to bring a new awareness of the negative effects due to stigma of mental illness. What NAMI did was long overdue and received unrelenting support from mental health professionals. Though a number of articles and a few books have appeared about the stigma of mental illness, perhaps this is the first book that clearly and comprehensively elucidates the prevalence of stigma, how much damage it does to individuals, and what specific strategies can be used to remove stigma at different levels.

This book-Challenging the Stigma of Mental Illness-has some distinctive qualities: Authored by a distinguished professor of Psychology from the Illinois Institute of Technology, an associate professor from the University of Haifa, and a professor at the Hong Kong Polytechnic University, this valuable book brings a more global, cosmopolitan approach to the problem of stigma of mental illness, because as the authors state, stigma is an international problem. For example, some Asians here in North America avoid seeking treatment for mental health problems, because they are scared of the stigma attached to mental illness. Long delays in seeking treatment tends to worsen the conditions.

The book is exceptional in other respects. It has a thorough and systematic approach to explain issues of stigma of mental illness from different viewpoints. First, the authors emphasize that stigma is personal, and they explain how to understand and measure stigma. The next two chapters, on chal- 
lenging the public stigma of mental illness and on fostering empowerment, are very interesting and informative. The last chapter on stigma and social injustice is especially enlightening.

This book is an important contribution, as it fills a gap in the mental health delivery system by reaching out to family, consumers, and providers. The breadth and depth of the coverage, and the combined wisdom of the experienced authors, make this book an ultimate resource on stigma of mental illness. The authors deserve praise for this work as it supersedes all earlier treatments. I expect that this book will be the definitive work for many years to come.

Dr. Basit is a former Editor-in-Chief of the Journal of Muslim Mental Health, has an international educational background and rich and varied experience in the field of mental health. He is currently a Senior Clinician and Director of Research at Orland Family Services, Orland Park, Illinois. 